45 (II09)

\title{
A height-weight formula to estimate the surface area of man.
}

\section{By Delafield Du Bois and E. F. Du Bois.}

[From the Russell Sage Institute of Pathology in Affiliation with the Second Medical Division, Bellevue Hospital.]

Using the method previously described before this society, ${ }^{1}$ it has been possible, with the aid of Miss Sawyer and Mr. Stone, to measure the surface area of a total of ro individuals. The "Linear Formula" previously described, when applied to these, gives an average error of $\mathbf{I} .5$ per cent. The chief limitation of the "Linear Formula" is that it involves the taking of 19 measurements. In the literature of the respiratory metabolism already published the only data given in regard to most of the subjects are the height and weight.

On the basis of the actual measurement of the surface of ro subjects of widely varying shape, formulas have been constructed on the plan of $A=W^{1 / a} \times H^{1 / b} \times C$, in which $A$ is the surface area in square cm., $W$ is the weight in kilograms and $H$ the height in $\mathrm{cm}$. and $C$ a constant. Various formulas were tried, care being taken that $3 / a+\mathrm{I} / b$ should always equal 2 in order that the formula might remain bi-dimensional. The formula $A=W^{1 / 3}$ $\times H^{1 / 1} \times C$ gave an average error of \pm 3.3 per cent. The formula $A=W^{1 / 2} \times H^{1 / 2} \times C$ gave an average error of 2.2 per cent. It was evident that values for $\mathrm{I} / a$ somewhere between $\mathrm{I} / 3$ and $\mathrm{I} / 2$ would give better results. By a rather lengthy process of calculation it was found that the average error could be reduced to $\mathrm{I} .7$ per cent. if the formula were made $A=W^{1 / 2.35} \times H^{1 / 1.38} \times C$. The calculation is not difficult if logarithms be used but a chart has been devised by means of which it is possible to estimate the surface area at a glance. The ordinates represent the height

Hospital Medical College, to whom we here express our sincere thanks. He found the macroscopic and microscopic evidences of emphysema, with the exception of increase in connective tissue. There were also areas resembling infarctions probably due to rhexis as a result of the increased blood pressure in the pulmonary circuit.

1 D. Du Bois and E. F. Du Bois, Proc. Soc. Exp. Biol. ANd MEd., I9I4, XII, I6; Arch. Int. Med., r915, XV, 868. 
in $\mathrm{cm}$., the abscissæ the weight in kilograms and curved lines drawn diagonally across the chart give the readings for the surface area in square meters. The details of the work with the chart and values of the constants will appear shortly in the Archives of Internal Medicine.

$$
46 \text { (III0) }
$$

\section{Diabetic dietetics. Glucose formation from protein foods.}

By N. W. JaNneY and F. A. Csonka (by invitation.).

[From the Chemical Laboratory of the Montefiore Home and Hospital, New York City.]

The carbohydrate content of foods has been usually accepted as a gauge of their adaptability to the dietary of diabetics. It has, however, been demonstrated that proteins yield in the glycosuric organism large amounts of glucose (50 to 80 per cent. of their weight, Janney). An exact knowledge of how much sugar arises in diabetic metabolism from protein food is therefore of some importance.

By observing certain precautions it was found possible to determine rather accurately the amounts of glucose yielded by various meats fed to dogs made completely diabetic by phlorizin. The sugar formed from beef, chicken, chicken eggs, rabbit and fish could thus be ascertained to represent from 9 to I 2 per cent. of the uncooked moist food. The solid substances of these materials produced from 36 to 48 per cent. glucose. Broiling and frying lead to considerable loss of water with corresponding increase of the percentage formation of glucose. Broiled beefsteak would yield $\mathbf{7} 7.5$ per cent. glucose.

Flour fed in preliminary experiments of the same nature gave rise to 92.5 per cent. of sugar on an anhydrous basis of calculation. From this data it was computed that in regard to sugar production or liberation in the organism $100 \mathrm{gm}$. bread is equivalent to about $350 \mathrm{gm}$. broiled beefsteak. In formulating diets for diabetics, glucose formation from the protein as well as the carbohydrate content of the food should therefore be considered. The experiments here alluded to will be later reported in detail. 